\title{
Exploring the Underlying Mechanisms of the Volume-Outcome Relationship in Complex Cancer Care
}

\author{
Ryan J. Ellis, MD ${ }^{1}$ and Ryan P. Merkow, MD, MS ${ }^{1,2}$ \\ ${ }^{1}$ Surgical Outcomes and Quality Improvement Center (SOQIC), Department of Surgery, Feinberg School of Medicine, \\ Northwestern University, Chicago, IL; ${ }^{2}$ Department of Surgery, Memorial Sloan Kettering Cancer Center, New York, NY
}

Over the past several decades, surgical volume status has become an established surrogate for complex cancer care quality. Both short- and long-term outcomes have been associated with hospital type (e.g. teaching status, Commission on Cancer designation) and case volume for a wide variety of cancers. ${ }^{1-3}$ The volume-outcome relationship has further been linked to more complex issues such as patient selection, guideline-recommended treatment, surgical technical quality, and case complexity. ${ }^{4,5}$ One of the primary challenges in interpreting this data is defining what specific aspects of care are the primary drivers of improved outcomes. For example, one 'spillover' association of the surgical volume-outcome relationship includes higher-volume specialists (e.g. medical and radiation oncologists) who are essential to the provision of high-quality cancer care. Because it is not feasible to funnel all complex cancer patients to high-volume centers, discerning which aspects of caretruly leads to improved outcomes at high-performing institutions is paramount to identifying modifiable factors that would be actionable for lower-performing hospitals. It is also important to emphasize that not all high- and low-volume centers are created equal. There are both high and low performers in each group. ${ }^{5}$

Among the most well-described, disease-modifying parameters in rectal cancer is the appropriate utilization of neoadjuvant chemoradiotherapy. ${ }^{6}$ In a subset of patients, neoadjuvant therapy can result in pathologic complete response (pCR), which is associated with reduced

(C) Society of Surgical Oncology 2017

First Received: 9 April 2017;

Published Online: 5 May 2017

R. P. Merkow, MD, MS

e-mail: merkowr@mskcc.org recurrence and improved overall survival. ${ }^{7}$ In this issue of Annals of Surgical Oncology, Lorimer et al. sought to identify predictors of $\mathrm{pCR}$ following neoadjuvant therapy through using the National Cancer Database. ${ }^{8}$ Their results demonstrated a consistent correlation between hospital surgical volume and improved $\mathrm{pCR}$ rates. While univariate analysis demonstrated several other patient- and tumorrelated factors associated with pCR, notably grade and T and $\mathrm{N}$ stage, the relationship between surgical volume and pCR was maintained on multivariable analysis. This is an interesting finding that could potentially explain an aspect of rectal cancer management underlying the volume-outcome relationship. Lorimer et al. accurately concluded that it remains difficult to determine what actionable drivers might lead to the observed difference in $\mathrm{pCR}$. They postulate several mechanisms, including an optimal timing between treatment and surgery and improved adherence to guideline recommendations at high-volume centers. These results certainly deserve further investigation.

The continued study of factors associated with realworld surgical quality would not be possible without cancer registries such as the Surveillance, Epidemiology and End Results (SEER) program and the National Cancer Database (NCDB). ${ }^{9}$ The NCDB captures more than $70 \%$ of cancer cases nationally, making it an ideal database for defining treatment trends, particularly the use of multimodality cancer care in the US. ${ }^{10,11}$ Moreover, the NCDB has allowed researchers the unique ability to investigate more nuanced questions, such as factors related to pCR.

However, cancer registries have several limitations that should be considered and were underscored by this study. ${ }^{12}$ The NCDB still lacks important surgical and oncologic data, such as recurrence, detailed tumor characteristics (e.g. distance from the anal verge), and specific chemotherapy regimens. More precise clinical indicators that guide treatment and influence outcomes, such as gene mutation 
status and detailed molecular analyses, are also generally not included in these databases.

Studies from the NCDB, such as the Lorimer et al. report, further underscore what must continue to be a focus of oncologic health services research: to automate the union of granular electronic health records with cancer registry data. This goal will become more attainable as hospitals continue to uniformly adopt electronic health record mandates. Full integration will allow for reanalysis of older data while incorporating important clinical variables as well as new diagnostic and therapeutic advancements in the field. This will give researchers the ability to perform 'deep dives' to investigate real world mechanisms underlying differences in patient selection, treatments and outcomes.

There is enormous potential in large datasets such as the NCDB. With more granular data and harnessing the full potential of the electronic medical record, additional improvements in the measurement of cancer care quality are most certainly on the horizon. In the meantime, it is important to recognize and commend the work of Lorimer et al. as we work towards identifying the true drivers of high-quality cancer care.

\section{REFERENCES}

1. Bilimoria KY, Bentrem DJ, Feinglass JM, et al. Directing surgical quality improvement initiatives: comparison of perioperative mortality and long-term survival for cancer surgery. J Clin Oncol. 2008;26(28):4626-33.
2. Schrag D, Panageas KS, Riedel E, et al. Hospital and surgeon procedure volume as predictors of outcome following rectal cancer resection. Ann Surg. 2002; 236(5):583-92.

3. Birkmeyer JD, Stukel TA, Siewers AE, et al. Surgeon volume and operative mortality in the United States. $N$ Engl $J$ Med. 2003;349(22):2117-27.

4. Merkow RP, Bilimoria KY, Bentrem DJ, et al. National assessment of margin status as a quality indicator after pancreatic cancer surgery. Ann Surg Oncol. 2014;21(4):1067-74.

5. Merkow RP, Bentrem DJ, Chung JW, et al. Differences in patients, surgical complexity, and outcomes after cancer surgery at National Cancer Institute-designated cancer centers compared to other hospitals. Med Care. 2013;51(7):606-13.

6. Fleming FJ, Pahlman L, Monson JR. Neoadjuvant therapy in rectal cancer. Dis Colon Rectum. 2011;54(7):901-12.

7. Martin ST, Heneghan HM, Winter DC. Systematic review and meta-analysis of outcomes following pathological complete response to neoadjuvant chemoradiotherapy for rectal cancer. $\mathrm{Br}$ J Surg. 2012;99(7):918-28.

8. Lorimer PD, et al. Pathologic complete response rates after neoadjuvant treatment in rectal cancer: an analysis of the NCDB. Ann Surg Oncol. 2017.

9. National Cancer Institute. SEER-Medicare publications by journal \& year. Updated 24 Feb 2017. https://healthcaredelivery. cancer.gov/seermedicare/overview/pubs_jour_year.php. Accessed 7 Apr 2017.

10. Mohanty S, Bilimoria KY. Comparing national cancer registries: the National Cancer Data Base (NCDB) and the Surveillance, Epidemiology, and End Results (SEER) program. J Surg Oncol. 2014;109(7):629-30.

11. Boffa DJ, Rosen JE, Mallin K, et al. Using the National Cancer Database for outcomes research: a review. JAMA Oncol. doi:10. 1001/jamaoncol.2016.6905.

12. Blanchard P, Garden AS. Looking beyond the numbers: highlighting the challenges of population-based studies in cancer research. J Clin Oncol. 2016;34(19):2317-8. 